\title{
A radiographic evaluation of facet sagittal angle in cervical spinal cord injury without major fracture or dislocation
}

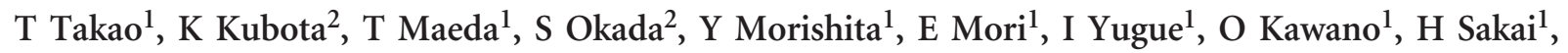 \\ T Ueta ${ }^{1}$ and K Shiba ${ }^{1}$
}

Study Design: A retrospective radiographic study with a minimum 2-year follow-up.

Objective: To evaluate the relationships between the cervical articular facets' morphology and the incidence of traumatic cervical spinal cord injury (CSCI) without major fracture or dislocation.

Setting: Spinal Injuries Center, Japan.

Methods: This study included 113 patients with traumatic CSCI without major fracture or dislocation. Eighty-four healthy volunteers without neurological deficits or cervical cord pathology on magnetic resonance imaging (MRI) were defined as control subjects. We used a plain sagittal radiograph to measure the facet sagittal angles (FSA) at four cervical segments in all the CSCI patients and controls. We defined the FSA as the angle between the inferior margin of the superior cervical spinal body and the inferior articular process of the superior vertebra.

Results: Most frequent incidence of CSCl was seen at C3-4 segment (54\%). With respect to CSCl at C3-4 segment, 55.7\% of the subjects showed smallest FSA at C3-4 segment.

Conclusion: Most of the traumatic CSCl at C3-4 segment showed raised cervical articular facets at C3-4 segment. On the basis of our results, we hypothesized that the raised cervical articular facets might have an important role in the etiology of traumatic CSCI. The cervical spinal cord at the C3-4 segment might receive the highest load during acute hyperextension of the cervical spine because of the C3-4 articular facets' morphology.

Spinal Cord (2017) 55, 515-517; doi:10.1038/sc.2016.172; published online 20 December 2016

\section{INTRODUCTION}

The incidence of traumatic cervical spinal cord injury (CSCI) without major fracture or dislocation has increased in developed countries because of the increasing elderly population. ${ }^{1}$ Most patients with CSCI are elderly and presented with spinal hyperextension predominantly at the C3-4 level. ${ }^{2}$ Several studies have reported that the cervical spinal cord at the C3-4 segment might receive the highest load during acute hyperextension of the cervical spine. ${ }^{3-5}$ We previously studied about the relationships between spinal canal diameter and pathophysiology of traumatic CSCI without major fracture or dislocation. ${ }^{6,7}$ In the study, the narrow spinal canal might be an important risk factor for the incidence of traumatic CSCI. However, we could not find the exact etiology why most of traumatic CSCI occurred at the C3-4 segment. To our knowledge, few reports, included in our previous study, have described the biomechanical etiology of traumatic CSCI without major fracture or dislocation, and this remains unclear.

Through our previous studies, we hypothesized that the development of traumatic CSCI without major fracture or dislocation was associated with cervical articular facets' morphology. In the study, we measured the cervical facet sagittal angle by using a plane radiograph in the sagittal plane. The aim of the present study was to evaluate the relationships between the cervical articular facets' morphology and the incidence of traumatic CSCI without major fracture or dislocation.

\section{MATERIALS AND METHODS}

\section{Study population}

From 2005 to 2011, 194 patients with traumatic CSCI without major fracture or dislocation were treated in our facility. All these patients underwent functional plain radiography, computed tomography, magnetic resonance imaging (MRI) on the cervical spine and neurological examination by a senior spine surgeon at the time of admission. All the subjects were admitted to our facility within 2 days of trauma, and had evidence of CSCI with cervical intramedullary intensity change on T2-weighted MRI. The following subjects were excluded from this study: patients with multiple segmental cervical cord injuries, existing cervical myelopathy before trauma, apparent herniated disc at the injured segment, severe instability at the injured segment on functional radiography, or ankylosing spondylitis at cervical spine.

In this study, 113 patients (101 men and 12 women; mean age, 62 years (range, 22-88 years)) with traumatic CSCI without major fracture or dislocation were included. Of these, 3 subjects had an injury at the C2-3 segment, 61 subjects had an injury at the C3-4, 32 subjects had an injury at the C4-5, 13 subjects had an injury at the C5-6 and 4 subjects had an injury at the C6-7. Eighty-four healthy volunteers (HV) (51 men and 33 women; mean age, 51 years (range, 28-86 years)) without neurological deficits or cervical cord pathology on MRI were defined as control subjects. No significant difference in age was found between CSCI and HV groups. The summaries of traumatic CSCI patients and HV are shown in Table 1.

Institutional review board approval was granted and informed consent was obtained from all of the patients.

${ }^{1}$ Department of Orthopaedic Surgery, Spinal Injuries Center, lizuka, Japan and ${ }^{2}$ Department of Orthopaedic Surgery, Kyushu University, Fukuoka, Japan Correspondence: Dr T Takao, Department of Orthopedic Surgery, Spinal Injuries Cente, 550-4 Igisu, lizuka 820-0053, Japan.

E-mail: tsuneaki@iris.ocn.ne.jp

Received 1 December 2015; revised 29 October 2016; accepted 30 October 2016; published online 20 December 2016 
Measurement of the facet sagittal angle in the sagittal plane

We used a plain radiograph in the sagittal plane to measure the facet sagittal angle (FSA) at four cervical segments (C3-4, C4-5, C5-6 and C6-7) in all of the CSCI patients and controls. We defined FSA as the angle between the inferior margin of the superior cervical spinal body and the inferior articular process of the superior vertebra (Figure 1).

\section{Statistical analysis}

The Mann-Whitney $U$ test and Fisher's exact test were used for statistical analyses. A $P$-value of $<0.05$ was considered statistically significant.

\section{RESULTS}

In the present series, the incidence rates of traumatic CSCI without major fracture or dislocation at the C2-3, C3-4, C4-5, C5-6 and C6-7 segments were $2.7,54,28.3,11.5$ and $3.5 \%$, respectively. Most frequent incidence of CSCI was seen at C3-4 segment.

The mean values of the FSA at the four cervical segments for the subjects with traumatic CSCI and HV are shown in Table 2. When compared with traumatic CSCI and HV, there were no significant differences at all of the segments. Among all the segments, except C6-7, the C3-4 segment had a significantly smaller FSA than the C4-5 and C5-6 segments in both groups. (Figures $2 \mathrm{a}$ and $\mathrm{b}$ ).

The relationships between the incidence of traumatic CSCI without major fracture or dislocation and the morphology of cervical articular facets are shown in Table 3. 55.7\% of the C3-4 SCI showed smallest

Table 1 The patients with traumatic cervical spinal cord injury (CSCI) and healthy volunteers (HV)

\begin{tabular}{lcc}
\hline & Traumatic CSCl & $H V$ \\
\hline No. (M:F) & $113(101: 12)$ & $84(51: 33)$ \\
Average age & $62(22-88)$ & $51(28-86)$
\end{tabular}

Abbreviations: $\mathrm{CSCl}$, cervical spinal cord injury; $\mathrm{HV}$, healthy volunteers.

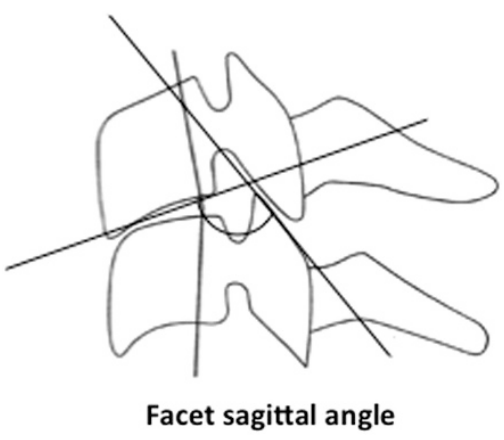

Figure 1 Facet sagittal angle (FSA). We defined the FSA as angle between the inferior margin of the superior cervical spinal body and the inferior articular process of the superior vertebrae.

FSA at the C3-4 segment. Moreover, with respect to C3-4 SCI, the Table 2 The average values of the FSA at the four cervical segments

\begin{tabular}{lcccc}
\hline & \multicolumn{5}{c}{ Facet sagittal angle $($ FSA $)\left({ }^{\circ}\right)$} \\
\cline { 2 - 5 } & C3-4 & C4-5 & C5-6 & C6-7 \\
\hline $\mathrm{CSCl}$ & $\begin{array}{c}133.4 \pm 6 \\
\mathrm{~ns}\end{array}$ & $\begin{array}{c}136 \pm 5.7 \\
\mathrm{~ns} \\
\mathrm{HV}\end{array}$ & $\begin{array}{c}135 \pm 6.2 \\
\mathrm{~ns}\end{array}$ & $\begin{array}{c}128.4 \pm 6.5 \\
\mathrm{~ns}\end{array}$ \\
\hline
\end{tabular}

Abbreviations: $\mathrm{CSCl}$, cervical spinal cord injury; $\mathrm{HV}$, healthy volunteers; ns, not significant. Mann-Whitney $U$ test was used for this statistical analysis.
C3-4 FSA was significantly smaller than those at the C4-5 and C5-6 segments $(P=0.0195$, Fisher's exact test $)$.

\section{DISCUSSION}

Several studies have reported the frequent incidence of traumatic CSCI at the level of C3-4 segment in the Japanese population. ${ }^{8}$ We believe that morphological differences between the cervical segments may contribute to the mechanism of traumatic CSCI. Hayashi et al., ${ }^{9}$ reported that the cervical spine of the older subjects displayed narrowing of intervertebral discs and osteophytes at the levels of C5-6 and C6-7, where range of motion was decreased. Such degenerative changes resulted in retrolisthesis predominantly at the levels of C3-4 and C4-5, where intervertebral disc space was well maintained and mobility was well preserved. Moreover, Koyanagi et al. ${ }^{10}$ hypothesized that the restricted intervertebral movement of the lower cervical segments due to degenerative changes might in fact protect the spinal cord at these segments from traumatic injury. Therefore, the upper segments (C3-4 or C4-5) rostral to the fixed segments might be damaged with cervical spinal hyperextension. However, through our experiences, not only elder patients but also many younger patients without degenerative changes in the lower cervical segments developed CSCI at the C3-4 segment. On the basis of this factor, we could not agree with their hypothesis. Imajo et al. ${ }^{4}$ reported that the C3-4 finite element model with $60^{\circ}$ facet was most susceptible to CSCI without radiological abnormality and that the bony pincers mechanism was dependent on facet joint inclination.

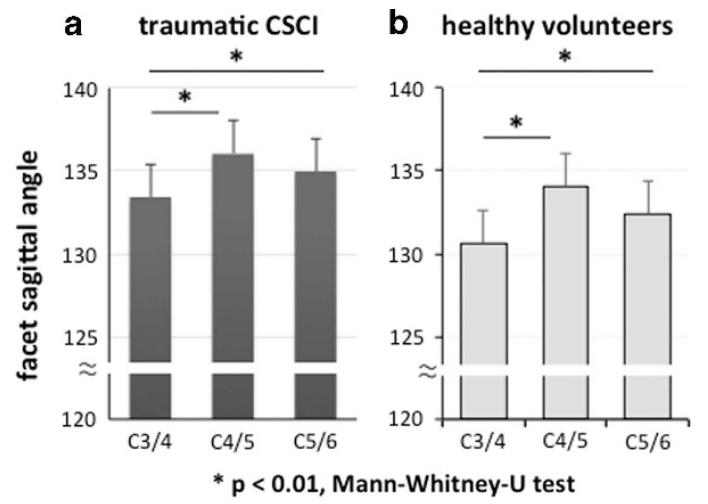

Figure 2 The FSA at the three cervical segments for the subjects with traumatic $\mathrm{CSCl}$ without major fracture or dislocation (a) and the healthy volunteers (b). Among all the segments, except C6-7, the C3-4 segment had a significantly smaller FSA than the C4-5 and C5-6 segments in both groups.

Table 3 The relationships between the incidence of traumatic $\mathrm{CSCl}$ without major fracture or dislocation and the morphology of cervical articular facets

\begin{tabular}{lcccc}
\hline \multirow{2}{*}{ Injured segment } & No. & \multicolumn{3}{c}{ Smallest FSA } \\
\cline { 3 - 5 } & & C3-4 & C4-5 & C5-6 \\
\hline C3-4 & 61 & $34(55.7 \%)$ & $11(18 \%)$ & $16(26.3 \%)$ \\
C4-5 & 31 & $13(41.9 \%)$ & $8(25.8 \%)$ & $10(32.3 \%)$ \\
C5-6 & 13 & $6(41.1 \%)$ & $2(15.4 \%)$ & $5(38.5 \%)$ \\
\hline
\end{tabular}

Abbreviation: FSA, facet sagittal angle. 
On the other hand, Morishita et al. ${ }^{5}$ reported that the cervical spinal cord at the C3-4 segment might receive the highest bony impingement load during acute hyperextension of the cervical spine. We previously studied about the relationships between cervical spinal canal diameter and the incidence of traumatic CSCI without major fracture or dislocation. ${ }^{6,7}$ However, we could not find the significant relationships between cervical spinal canal diameter and the incidence of traumatic CSCI without major fracture or dislocation. Those published papers could not indicate the exact biomechanical etiology of traumatic CSCI without major fracture or dislocation.

The FSA at the C3-4 segment demonstrated significant smaller angle when compared with the C4-5 and C5-6 segments. Moreover, most of traumatic CSCI at the C3-4 segment showed smallest FSA at the C3-4 segment. On the basis of our results, we hypothesized that the raised cervical articular facets might have an important role in the etiology of traumatic CSCI without major fracture or dislocation. The cervical spinal cord at the C3-4 segment might receive the highest mechanical stress during acute hyperextension of the cervical spine because of the C3-4 articular facets' morphology.

Some issues remain unaddressed in this study. In the study, the C6-7 FSA was smallest among the cervical segments. However, we could not discuss the affects of C6-7 articular facets' morphology on the etiology of traumatic CSCI. Using the present investigation as the pilot study, further research that uses anatomical analysis of the cervical spinal column with a larger patient population may help shed light on these issues. Moreover, the biomechanical etiology of traumatic CSCI without major fracture or dislocation should be clarified in quite some detail.

\section{DATA ARCHIVING}

There were no data to deposit.

\section{CONFLICT OF INTEREST}

The authors declare no conflict of interest.

1 Kawano O, Ueta T, Shiba K, Iwamoto Y. Outcome of decompression surgery for cervical spinal cord injury without bone and disc injury in patients with spinal cord compression: a multicenter prospective study. Spinal Cord 2010; 48: 548-553.

2 Okada S, Maeda T, Ohkawa Y, Harimaya K, Saiwai H, Kumamaru H et al. Does ossification of the posterior longitudinal ligament affect the neurological outcome after traumatic cervical cord injury? Spine 2009; 34: 1148-1152.

3 Ueta T. Cervical cord injuries without X-ray evidence of bony damages-Its pathology and treatment in the early stage. Nitidoku Ihou (in Japanese) 2000; 45: 301-316.

4 Imajo Y, Hiiragi I, Kato Y, Taguchi T. Use of the finite element method to study the mechanism of spinal cord injury without radiological abnormality in the cervical spine. Spine 2009; 34: E83-E87.

5 Morishita Y, Naito M, Wang JC. Cervical spinal canal stenosis: the differences between stenosis at the lower cervical and multiple segment levels. Int Orthop 2011; 35: 1517-1522.

6 Takao T, Morishita Y, Okada S, Maeda T, Katoh F, Ueta T et al. Clinical relationship between cervical spinal canal stenosis and traumatic cervical spinal cord injury without major fracture or dislocation. Eur Spine J 2013; 22: 2228-2231.

7 Takao T, Okada S, Morishita Y, Maeda T, Kubota K, Ideta R et al. The clinical influence of cervical spinal canal stenosis on the neurological outcome after traumatic cervical spinal cord injury without major fracture or dislocation. Asian Spine J 2016; 10: 536-542.

8 Shimada K, Tokioka T. Sequential MRI studies in patients with cervical cord injury but without bony injury. Paraplegia 1995; 33: 573-578.

9 Hayashi H, Okada K, Hamada M, Toda K, Ueno R. Etiologic factors of myelopathy. a radiographic evaluation of the aging changes in the cervical spine. Clin Orthop Relat Res 1987; 214: 200-209.

10 Koyanagi I, Iwasaki Y, Hida K, Akino M, Imamura H, Abe $\mathrm{H}$ et al. Acute cervical cord injury without fracture or dislocation of the spinal column. J Neurosurgery 2000; 93 : 15-20. 